\title{
Matched witness for multipartite entanglement
}

\author{
Xiao-yu Chen ${ }^{a *}$ Zhu-an $\mathrm{Xu}^{b}$ \\ ${ }^{a}$ College of Information and Electronic Engineering, Zhejiang Gongshang University, Hangzhou, Zhejiang 310018, China \\ ${ }^{b}$ Department of Physics, Zhejiang University, Hangzhou, Zhejiang 310027, China
}

\section{Abstract}

We transform the way of finding entanglement criterion into two steps: to obtain necessary criterion of separability by maximizing an algebra function for a set of characteristic variables of the witness operator and the given number of partitions, then to obtain the sufficient criterion by minimizing an algebra function with respect to the characteristic variables for a given quantum state. Our method avoids the semi-definite program calculation in the witness operator entanglement detection. The necessary and sufficient criterion of separability for the three qubit X shaped state is given to illustrate the procedure of finding the criterion. We give the necessary and sufficient criteria of the three partite and full separabilities for the four qubit noisy GHZ state and the four qubit noisy cluster state.

PACS number(s): 03.67.Mn; 03.65.Ud

Keywords: Multipartite entanglement; Separable criterion; Cluster state; GHZ state

\section{Introduction}

Quantum entanglement is considered as the central resource in quantum communication and quantum computation. It is a special form of bipartite or multipartite quantum superposition, and intrinsically different from any classical system for its structures and properties. Closely related to entanglement is the separable state. A separable state is the state which can be written as the probability mixture of product states [1. An entangled state is simply not separable. To determine a given state is entangled or not is still very difficult in general now. Thus entanglement criteria are desirable. Many progresses have been achieved for the criteria of entanglement or separability. Among them are the Peres-Horodecki criterion [2] 3], the computable cross norm 4 or realignment criterion [5], the entropy criterion, the uncertainty criterion [6] and so on 7 [8]. All these criteria are necessary conditions for separability. Violation of them means entanglement. The criteria are mainly applied to bipartite states, the criteria for multipartite entanglement are sporadic [9, 10]. One the other

*Email:xychen@zjgsu.edu.cn hand, entanglement witness is also a mean for entanglement detection 11, especially friendly for experiment. In this paper, we will start from the original definition of separable state, combine with entanglement witness method to derive multipartite entanglement criteria. We find that the process in finding the necessary criteria may also give rise to the sufficient criteria of separability.

\section{Entanglement witness in charac- teristic form}

A multipartite state $\rho$ is separable when it can be written as [1]

$$
\rho=\sum_{i} p_{i} \rho_{i}^{A_{1}} \otimes \rho_{i}^{A_{2}} \otimes \ldots \otimes \rho_{i}^{A_{N}}
$$

where $\rho_{i}^{A_{j}}$ is the state (it is always possible to assume it to be pure) of $A_{j}$ part, $p_{i}$ form a probability distribution. The method of partition of the system into $N$ parts may change with the index $i$ to cope with the concepts of biseparable, $k$-separable and fully separable. In the following, we will consider the three qubit system. Extending to multipartite qudit system is straightforward. For the problem of full separability of a three qubit state $\rho$, its characteristic function is

$$
R_{i j k}=\operatorname{tr}\left[\rho\left(\sigma_{i} \otimes \sigma_{j} \otimes \sigma_{k}\right)\right],
$$

where $i, j, k=0,1,2,3 ; \sigma_{i}$ are Pauli matrices for $i=1,2,3$ and $\sigma_{0}=I_{2}$ is the $2 \times 2$ identity matrix. Let the characteristic function of an entanglement witness $W$ be $-M_{i j k}$ (We call $M_{i j k}$ characteristic variables). We set $M_{i j k}$ to be zero when $R_{i j k}$ is zero for simplicity. A state $\rho$ is entangled when

$$
-\operatorname{tr}(\rho W)=8 \sum_{i j k} R_{i j k} M_{i j k}>0,
$$

while for all fully separable states $\rho^{s}$ we have

$$
-\operatorname{tr}\left(\rho^{s} W\right)=8 \sum_{i j k} R_{i j k}^{s} M_{i j k} \leq 0 .
$$

The problem then is reduced to find a series of parameters $M_{i j k}$. For a three particle fully separable state $\rho$, its characteristic function is $R_{i j k}^{s}=\sum_{l} p_{l} x_{i l} y_{j l} z_{k l}$, where $x_{i l}=\operatorname{tr}\left(\rho_{l}^{A_{1}} \sigma_{i}\right)$, with $x_{0 l}=1$, unit (Bloch) vector 
$\mathbf{x}_{l}=\left(x_{1 l}, x_{2 l}, x_{3 l}\right)$ (since $\rho_{l}^{A_{1}}$ is pure) and similar for $y_{j l}, z_{k l}$. Notice that for any state $R_{000}=1$, then $\sum_{i j k} R_{i j k}^{s} M_{i j k}=$ $\sum_{l} p_{l} x_{i l} y_{j l} z_{k l} M_{i j k}=\sum_{l}^{\prime} p_{l} x_{i l} y_{j l} z_{k l} M_{i j k}+M_{000} \leq 0$, where the prime in the summation means that the $i=j=k=0$ term is removed from the summation. We define $M_{000} \equiv-\max _{\mathbf{x}, \mathbf{y}, \mathbf{z}} \sum_{i j k}^{\prime} M_{i j k} x_{i} y_{j} z_{k}$ with $x_{0}=y_{0}=z_{0}=1,|\mathbf{x}|=|\mathbf{y}|=|\mathbf{z}|=1$. Such a definition leads to a legal entanglement witness in the sense that its keeps $\operatorname{tr}(\rho W) \geq 0$ for all fully separable states. For given parameters $M_{i j k}$ with $M_{000}$ yet to be determined, the maximization $\max _{\mathbf{x}, \mathbf{y}, \mathbf{z}} \sum_{i j k}^{\prime} M_{i j k} x_{i} y_{j} z_{k}$ can be carried out analytically or numerically. Denote vectors $\mathbf{M}=\left(M_{001}, M_{002}, M_{003}, \ldots, M_{333}\right) \quad$ and $\mathbf{R}=\left(R_{001}, R_{002}, R_{003}, \ldots, R_{333}\right), \quad$ then the necessary condition of full separability is

$$
\mathbf{M} \cdot \mathbf{R} \leq-M_{000} .
$$

Violation of it implies entanglement. To find the matched witness, we calculate the following minimization,

$$
p=\min _{\mathbf{M}} \frac{-M_{000}}{\mathbf{M} \cdot \mathbf{R}} .
$$

Keep in mind that $\mathbf{M}$ is so chosen such that $\mathbf{M} \cdot \mathbf{R}$ is positive, $M_{000}$ is negative. $1-p$ is the white noise tolerance of an entangled state $\rho$. If the state is fully separable, then $p \geq 1$.

For the problem of biseparability of a three qubit state, we first consider the characteristic function of a pure two qubit state $|\psi\rangle=\sum_{m, n=0}^{1} \alpha_{m n}|m n\rangle$. The characteristic function of $|\psi\rangle$ is denoted as $T_{i j}=\left\langle\psi\left|\sigma_{i} \otimes \sigma_{j}\right| \psi\right\rangle$. A state $\rho^{b s}$ is biseparable if

$$
\begin{aligned}
\rho^{b s}= & \sum_{l}\left(p_{1 l} \rho_{l}^{A_{1}} \otimes \rho_{l}^{A_{2} A_{3}}+p_{2 l} \rho_{l}^{A_{2}} \otimes \rho_{l}^{A_{1} A_{3}}\right. \\
& \left.+p_{3 l} \rho_{l}^{A_{3}} \otimes \rho_{l}^{A_{1} A_{2}}\right),
\end{aligned}
$$

where $\rho_{l}^{A_{m} A_{n}}$ is a pure two qubit state. The genuine entanglement witness $W$ with characteristic function $-M_{i j k}$ should fulfill the condition $-\operatorname{tr}\left(\rho^{b s} W\right)=8 \sum_{i j k} R_{i j k}^{b s} M_{i j k} \leq 0$ for any biseparable state $\rho^{b s}$. The characteristic function of $\rho^{b s}$ is $R_{i j k}^{b s}=\sum_{l} \sum_{i j k}\left(p_{1 l} x_{i l} T_{j k}^{(23)}+p_{2 l} y_{i l} T_{j k}^{(13)}+p_{3 l} z_{i l} T_{j k}^{(12)}\right)$.

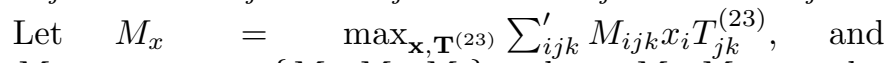
$M_{000}=-\max \left\{M_{x}, M_{y}, M_{z}\right\}$, where $M_{y}, M_{z}$ are defined similarly as $M_{x}$. The necessary condition of biseparability is still described by inequality (5), with a new definition of $M_{000}$. The noise tolerance of a genuine entangled state is $1-p$ too, with a different $p$ from a different definition of $M_{000}$.

\section{$3 \quad$ Three qubit $X$ shaped states}

The density matrix of a three qubit $X$ shaped state is a $8 \times 8$ matrix with diagonal entries, anti-diagonal entries and all the other entries are zero. The possible nonzero entries are denoted as $\rho_{00}, \rho_{11}, \ldots, \rho_{77}$ for diagonal elements and $\rho_{07}, \rho_{16}, \ldots, \rho_{70}$ for anti-diagonal elements. The decimal subscript $l$ is equivalent to the three bit binary string $l_{1} l_{2} l_{3}$ such that $l=4 l_{1}+2 l_{2}+l_{3}$. Hence we may write $\rho_{16}$ as $\rho_{001,110}$ in three bit binary subscripts. For an three qubit $X$ state, the nonzero elements of the characteristic function are $R_{i j k}$ with the subscripts $i, j, k=$ 0,3 or $i, j, k=1,2$. The unit vector $\mathbf{x}$ can be written as $\mathbf{x}=\left(\sin \theta_{1} \cos \varphi_{1}, \sin \theta_{1} \sin \varphi_{1}, \cos \theta_{1}\right)$ with $\theta_{1} \in[0, \pi]$, $\varphi_{1} \in[0,2 \pi]$. Similarly, $\mathbf{y}, \mathbf{z}$ are expressed with $\theta_{2}, \varphi_{2}$ and $\theta_{3}, \varphi_{3}$, respectively. Then $-M_{000}=\max _{\theta, \varphi} F(\theta, \varphi)$, with

$$
F(\theta, \varphi)=f(\theta)+s_{1} s_{2} s_{3} g(\varphi),
$$

and $\theta=\left(\theta_{1}, \theta_{2}, \theta_{3}\right), \varphi=\left(\varphi_{1}, \varphi_{2}, \varphi_{3}\right)$, where $f(\theta)=$ $M_{003} c_{3}+M_{030} c_{2}+M_{300} c_{1}+M_{033} c_{2} c_{3}+M_{303} c_{1} c_{3}+$ $M_{330} c_{1} c_{2}+M_{333} c_{3} c_{2} c_{1}$, we denote $\cos \theta_{i}=c_{i}, \sin \theta_{i}=s_{i}$ for short, and

$$
\begin{aligned}
g(\varphi)= & M_{111} c_{1}^{\prime} c_{2}^{\prime} c_{3}^{\prime}+M_{122} c_{1}^{\prime} s_{2}^{\prime} s_{3}^{\prime} \\
& +M_{212} s_{1}^{\prime} c_{2}^{\prime} s_{3}^{\prime}+M_{221} s_{1}^{\prime} s_{2}^{\prime} c_{3}^{\prime} .
\end{aligned}
$$

for the case of real anti-diagonal entries of an $X$ shaped state. Where $c_{i}^{\prime}=\cos \varphi_{i}, s_{i}^{\prime}=\sin \varphi_{i}$.

Lemma 1 Denote the maximum of $g(\varphi)$ in (8) with respect to $\varphi$ as $g_{m}$, then

$g_{m}=\left\{\begin{array}{c}\sqrt{\frac{(\delta \alpha+\beta \gamma)(\delta \beta+\alpha \gamma)(\delta \gamma+\alpha \beta)}{\delta \alpha \beta \gamma}}, \text { for } \delta \alpha \beta \gamma>0 \text { and } q \geq 0 \\ \max \left\{\left|M_{111}\right|,\left|M_{122}\right|,\left|M_{212}\right|,\left|M_{221}\right|\right\}, \text { otherwise. }\end{array}\right.$

Where $(\delta, \alpha, \beta, \gamma)=\frac{1}{4}\left(M_{111}, M_{122}, M_{212}, M_{221}\right) \Gamma$, here $\Gamma$ is a $4 \times 4$ matrix with all of its diagonal entries being -1 and off-diagonal entries being $+1 . q=q_{0} q_{1} q_{2} q_{3}$ with $\left(q_{0}, q_{1}, q_{2}, q_{3}\right)=(\alpha \beta \gamma, \delta \beta \gamma, \delta \alpha \gamma, \delta \alpha \beta) \Gamma$.

The proof can be found in Appendix.

Theorem 1 An $X$ shaped state with identical diagonal entries and real anti-diagonal entries is separable iff

$$
R \leq 1,
$$

where

$$
R=\left\{\begin{array}{c}
\sqrt{\frac{\left(R_{0} R_{1}+R_{2} R_{3}\right)\left(R_{0} R_{2}+R_{1} R_{3}\right)\left(R_{0} R_{3}+R_{1} R_{2}\right)}{R_{0} R_{1} R_{2} R_{3}}}, \\
\text { for } Q>0 \text { and } r \geq 0 \\
8 \max \left\{\left|\rho_{07}\right|,\left|\rho_{16}\right|,\left|\rho_{25}\right|,\left|\rho_{34}\right|\right\}, \text { otherwise }
\end{array}\right.
$$

with $R_{0}=R_{111}, R_{1}=R_{122}, R_{2}=R_{212}, R_{3}=R_{221}$ and $Q=R_{0} R_{1} R_{2} R_{3}$. Here $r=r_{0} r_{1} r_{2} r_{3}$ and vector $\left(r_{0}, r_{1}, r_{2}, r_{3}\right)=\left(R_{1} R_{2} R_{3}, R_{0} R_{2} R_{3}, R_{0} R_{1} R_{3}, R_{0} R_{1} R_{2}\right) \Gamma$.

Proof: "Only if": At present case, we have $-M_{000}=$ $g_{m}$ by assuming all the $M_{i j k}$ be 0 except $M_{000}$ and $M_{111}, M_{122}, M_{212}, M_{221}$. 
In (9), if $g_{m}=\max \left\{\left|M_{111}\right|,\left|M_{122}\right|,\left|M_{212}\right|,\left|M_{221}\right|\right\}$, let $\left|M_{111}\right|$ is the biggest w.l.o.g. The necessary condition (5) is

$$
L \equiv M_{111} R_{0}+M_{122} R_{1}+M_{212} R_{2}+M_{221} R_{3} \leq\left|M_{111}\right| \text {. }
$$

We may choose the signs of $M_{111}, M_{122}, M_{212}, M_{221}$ match the signs of $R_{i}(i=0, \ldots, 3)$ to make $L$ larger. If $Q<0$, then one or three $R_{i}$ are negative. Let $R_{0}$ be negative and the other $R_{i}$ be positive for definite. We may further require $\left|M_{122}\right|=\left|M_{212}\right|=\left|M_{221}\right|=\left|M_{111}\right|$ in order to make the left side of (11) even larger. This is possible if we choose $\alpha=\beta=\gamma=0$. For we obtain $\delta\left(-R_{0}+R_{1}+R_{2}+R_{3}\right) \leq|\delta|$. Thus $8\left|\rho_{07}\right| \leq 1$, and there are similarly inequalities for the other anti-diagonal entries. We then have $8 \max \left\{\left|\rho_{07}\right|,\left|\rho_{16}\right|,\left|\rho_{25}\right|,\left|\rho_{34}\right|\right\} \leq 1$. When $\delta \alpha \beta \gamma<0$, let $\left|\rho_{07}\right|$ be the largest one among $\left\{\left|\rho_{07}\right|,\left|\rho_{16}\right|,\left|\rho_{25}\right|,\left|\rho_{34}\right|\right\}$. If $\rho_{07}>0$, we may choose $\delta<0$ and $\alpha, \beta, \gamma>0$, we have $\frac{L}{g_{m}}-8 \rho_{07} \leq 0$, the equality is achieved when $|\delta| \rightarrow \infty$. If $\rho_{07}<0$, we may choose $\delta, \alpha, \beta>0$ and $\gamma<0$, we have $\frac{L}{a_{m}}+8 \rho_{07} \leq 0$, the equality is achieved when $\delta \rightarrow \infty$. Thus we have proved that $R=\max _{\mathbf{M}} \frac{L}{g_{m}}=8 \max \left\{\left|\rho_{07}\right|,\left|\rho_{16}\right|,\left|\rho_{25}\right|,\left|\rho_{34}\right|\right\}$ for the cases of $Q<0$ or $\delta \alpha \beta \gamma<0$.

If $g_{m}=\sqrt{(\delta \alpha+\beta \gamma)(\delta \beta+\alpha \gamma)(\delta \gamma+\alpha \beta) /(\delta \alpha \beta \gamma)}$, we may rewrite equation (6) as $p=\min _{\delta, \alpha, \beta, \gamma} \frac{g_{m}}{L}$, where $L=8\left(-\delta \rho_{07}+\gamma \rho_{16}+\beta \rho_{25}+\alpha \rho_{34}\right)$ is assumed to be positive. Then $\frac{\partial p}{\partial \delta}=0$ leads to $\frac{\partial \ln g_{m}}{\partial \delta}=\frac{\partial \ln L}{\partial \delta}$, which is $-8 \rho_{07}=\frac{L}{2}\left(\frac{\alpha}{\delta \alpha+\beta \gamma}+\frac{\beta}{\delta \beta+\alpha \gamma}+\frac{\gamma}{\delta \gamma+\alpha \beta}-\frac{1}{\delta}\right)$, similarly, $\rho_{16}, \rho_{25}, \rho_{34}$ can also be obtained. Then after some algebra we have $R_{0}=G L q_{1} q_{2} q_{3}, R_{1}=G L q_{0} q_{2} q_{3}, R_{2}=G L q_{0} q_{1} q_{3}$, $R_{3}=G L q_{0} q_{1} q_{2}$, with $G=\frac{1}{8 g_{m}^{2}(\delta \alpha \beta \gamma)^{2}}$. The first line of the right hand side of (10) can be written as

$$
R=G L \sqrt{\left(q_{0} q_{1}+q_{2} q_{3}\right)\left(q_{0} q_{2}+q_{1} q_{3}\right)\left(q_{0} q_{3}+q_{1} q_{2}\right)},
$$

Note that $q_{0} q_{1}+q_{2} q_{3}=4 \delta \alpha \beta \gamma(\delta \alpha+\beta \gamma)$, we arrive at $R=\frac{L}{g_{m}}$. Thus the noise tolerance of the state is $p=\frac{1}{R}$. The state is fully separable when $R \leq 1$. The condition $Q>0$ guarantees the validation of the first line of (10). Since $Q=G^{4} L^{4} q^{3}$, so $Q \geq 0$ is equivalent to $q \geq 0$. Also we have $r=\left(4 q^{2} G^{3} L^{3}\right)^{4}(\delta \alpha \beta \gamma)^{3}$, hence $r>0$ is equivalent to $\delta \alpha \beta \gamma>0$.

"If": In the case of $Q>0$ (and $r>0$ ), that the state is fully separable when $R \leq 1$ is shown in 12 [13 [14. In the coordinate of $\left(\rho_{07}, \rho_{16}, \rho_{25}, \rho_{34}\right)$, the shape of the fully separable state set is as follow: consider a four dimensional hypercube centered at original and with side length $1 / 4$, the 16 vertices are located at $\left|\rho_{07}\right|=\left|\rho_{16}\right|=\left|\rho_{25}\right|=\left|\rho_{34}\right|$ $=\frac{1}{8}$. The vertex $\left(\rho_{07}, \rho_{16}, \rho_{25}, \rho_{34}\right)=\left(\frac{1}{8}, \frac{1}{8}, \frac{1}{8}, \frac{1}{8}\right)$ corresponds to the state $\frac{1}{8}\left(I I I+\sigma_{1} \sigma_{1} \sigma_{1}\right)$, which is fully separable. The vertices with two or four anti-diagonal elements being $-\frac{1}{8}$ corresponds to states which are local equivalent to the fully separable state $\frac{1}{8}\left(I I I+\sigma_{1} \sigma_{1} \sigma_{1}\right)$. The vertex $\left(\rho_{07}, \rho_{16}, \rho_{25}, \rho_{34}\right)=\left(\frac{1}{8}, \frac{1}{8}, \frac{1}{8},-\frac{1}{8}\right)$ corresponds to an entangled state. The corner containing this vertex is cut by the hyper surface $R=1$ where $R$ is the first line of (10). Similarly, the other 7 corners with vertices which have odd number of $-\frac{1}{8}$ coordinate components are also cut. Thus the fully separable state set is a four dimensional hypercube with 8 of its corners cut. The 8 vertex states with even number of $-\frac{1}{8}$ coordinate components and the states in the cut surfaces are fully separable. This guarantees all the states correspond to the inner and surface points in the cut hypercube is fully separable.

Denote $F_{1}(\theta)=\max _{\varphi} F(\theta, \varphi)=f(\theta)+g_{m} s_{1} s_{2} s_{3}$, and the maximum of $F_{1}(\theta)$ with respect to $\theta_{3}$ as $F_{2}\left(\theta_{1}, \theta_{2}\right)$, then

$$
F_{2}\left(\theta_{1}, \theta_{2}\right)=a+b c_{2}+\sqrt{\left(c+d c_{2}\right)^{2}+e^{2} s_{2}^{2}} .
$$

Where $a=M_{300} c_{1}, b=M_{030}+M_{330} c_{1}, c=M_{003}+M_{303} c_{1}$, $d=M_{033}+M_{333} c_{1}, e=g_{m} s_{1}$.

We consider two cases. Case (i), let us assume $e^{2}=$ $d^{2}-c^{2}$, which requires $M_{033}=M_{330}, M_{030}=M_{333}$ (or $\left.M_{033}=-M_{330}, M_{030}=-M_{333}\right), M_{033}^{2}=M_{030}^{2}+g_{m}^{2}$. Then (13) is $F_{2}\left(\theta_{1}, \theta_{2}\right)=a+b c_{2}+\left|d+c c_{2}\right|$. Assuming $d+c c_{2}<$ 0 , then $F_{2}\left(\theta_{1}, \theta_{2}\right)=a+b c_{2}-d-c c_{2}$. Let $b=c$, that is $M_{030}=M_{003}, M_{330}=M_{303}$, then $F_{2}\left(\theta_{1}, \theta_{2}\right)=a-d$. If we further assume $M_{300}=M_{333}$, then $F_{2}\left(\theta_{1}, \theta_{2}\right)=-M_{033}$ is a constant. Hence if we choose the set of parameters as $M_{030}=M_{333}=M_{300}=M_{003}=\sin \eta, g_{m}=\cos \eta, M_{033}=$ $M_{330}=M_{303}=-1$, then all the assumptions $e^{2}=d^{2}-c^{2}$, $b=c, d+c c_{2}<0$ can be fulfilled. The necessary condition of full separability (5) reads

$$
4 \sin \eta\left(\rho_{00}-\rho_{77}\right)+R \cos \eta \leq 4\left(\rho_{00}+\rho_{77}\right)
$$

for any $\eta$. We have used $R_{003}+R_{030}+R_{300}+R_{333}=4\left(\rho_{00}-\right.$ $\left.\rho_{77}\right)$ and $1+R_{033}+R_{303}+R_{330}=4\left(\rho_{00}+\rho_{77}\right)$. The necessary condition can be refined to $\sqrt{16\left(\rho_{00}-\rho_{77}\right)^{2}+R^{2}} \leq$ $4\left(\rho_{00}+\rho_{77}\right)$, which is $\frac{1}{8} R \leq \sqrt{\rho_{00} \rho_{77}}$. Similarly, we obtain $\frac{1}{8} R \leq \sqrt{\rho_{33} \rho_{44}}$ by choosing $M_{033}=-M_{330}, M_{030}=$ $-M_{333}$ instead. If we properly choosing the parameters such that $e^{2}=d^{2}-c^{2}, b=-c, d+c c_{2}>0$, then we arrive at the necessary conditions of $\frac{1}{8} R \leq \sqrt{\rho_{22} \rho_{55}}$ and $\frac{1}{8} R \leq \sqrt{\rho_{11} \rho_{66}}$. Hence we have the necessary condition

$$
\frac{1}{8} R \leq \min _{i=0, \ldots, 3} \sqrt{\rho_{i i} \rho_{7-i, 7-i}} .
$$

Case (ii), let us assume $M_{033}=M_{300} \equiv M_{1}, M_{303}=$ $M_{030} \equiv M_{2}, M_{003}=M_{330} \equiv M_{3}, M_{333} \equiv M_{0}$ and $g_{m}=\sqrt[4]{m_{0} m_{1} m_{2} m_{3}}$, with the vector $\left(m_{0}, m_{1}, m_{2}, m_{3}\right)$ $=-\frac{1}{2} H\left(M_{0}, M_{2}, M_{1}, M_{3}\right)$. Here $H$ is the $4 \times 4$ Hadamard matrix. Let $M_{0}$ be negative and $-M_{0}$ be sufficiently large. Then we can prove that

$$
M_{000}=M_{333}=M_{0},
$$

see Appendix for details. Hence the necessary condition of full separability is

$$
\frac{1}{8} g_{m} R \leq m_{0} \rho_{00}+m_{1} \rho_{33}+m_{2} \rho_{55}+m_{3} \rho_{66},
$$


which is true for all possible choices of $m_{i} \quad(i=$ $0, \ldots, 3)$. Notice that $m_{0} \rho_{00}+m_{1} \rho_{33}+m_{2} \rho_{55}+m_{3} \rho_{66} \geq$ $4 \sqrt[4]{m_{0} m_{1} m_{2} m_{3}} \sqrt[4]{\rho_{00} \rho_{33} \rho_{55} \rho_{66}}$, the identity is achieved when

$$
m_{0} \rho_{00}=m_{1} \rho_{33}=m_{2} \rho_{55}=m_{3} \rho_{66} .
$$

Thus the condition (17) can be refined as $\sqrt[4]{\rho_{00} \rho_{33} \rho_{55} \rho_{66}} \geq$ $\frac{1}{8} R$. Similarly we have $\sqrt[4]{\rho_{11} \rho_{22} \rho_{44} \rho_{77}} \geq \frac{1}{8} R$. Hence the necessary condition of full separability is refined as

$$
\min \left(\sqrt[4]{\rho_{00} \rho_{33} \rho_{55} \rho_{66}}, \sqrt[4]{\rho_{11} \rho_{22} \rho_{44} \rho_{77}}\right) \geq \frac{1}{8} R .
$$

Here the role of matched witness is clearly shown by (18).

Theorem 2 A three qubit $X$ shaped state with real antidiagonal entries is fully separable iff

$\min _{i=0, \ldots, 3}\left(\sqrt[4]{\rho_{00} \rho_{33} \rho_{55} \rho_{66}}, \sqrt[4]{\rho_{11} \rho_{22} \rho_{44} \rho_{77}}, \sqrt{\rho_{i i} \rho_{7-i, 7-i}}\right) \geq \frac{1}{8} R$.

Proof: The "only if" comes from (15) and (19). For the "if " part, consider the operator identity $A_{1} A_{2} A_{3}+$ $B_{1} B_{2} B_{3}=\frac{1}{4}\left[\left(A_{1}+B_{1}\right)\left(A_{2}+B_{2}\right)\left(A_{3}+B_{3}\right)+\left(A_{1}+\right.\right.$ $\left.B_{1}\right)\left(A_{2}-B_{2}\right)\left(A_{3}-B_{3}\right)+\left(A_{1}-B_{1}\right)\left(A_{2}+B_{2}\right)\left(A_{3}-\right.$ $\left.\left.B_{3}\right)+\left(A_{1}-B_{1}\right)\left(A_{2}-B_{2}\right)\left(A_{3}+B_{3}\right)\right]$, let $A_{i}=I+$ $\cos \theta_{i} \sigma_{3}, B_{i}=\sin \theta_{i}\left(\cos \varphi_{i} \sigma_{1}+\sin \varphi_{i} \sigma_{2}\right)$, then the state $\frac{1}{8}\left(A_{1} A_{2} A_{3}+B_{1} B_{2} B_{3}\right)$ is fully separable for any $\theta_{i}$ and $\varphi_{i}$. Let $B_{i}^{\prime}=\sin \theta_{i}\left(\cos \varphi_{i} \sigma_{1}-\sin \varphi_{i} \sigma_{2}\right)$, then the state $\varrho=\frac{1}{16}\left(2 A_{1} A_{2} A_{3}+B_{1} B_{2} B_{3}+B_{1}^{\prime} B_{2}^{\prime} B_{3}^{\prime}\right)$ is a fully separable $X$ shaped state with real anti-diagonal entries. The anti-diagonal entries have been treated in Theorem 1, we have $R=\left|\sin \theta_{1} \sin \theta_{2} \sin \theta_{3}\right|$ for the fully separable state $\varrho$. In the case that all the terms in the bracket of the left hand side of (20) are equal, it is always possible to choose proper $\theta_{i}$ such that the state $\rho$ in (20) is equal to the fully separable state $\varrho$. If some of the terms in bracket of the left hand side of (20) are not equal with each other, then we have $\rho=(1-\kappa) \rho_{d}+\kappa \varrho$, with $0 \leq \kappa<1$ and $\rho_{d}$ is a diagonal state in computational basis thus fully separable.

\section{The tripartite separability of noisy four qubit GHZ state}

The four qubit GHZ state $\left|G H Z_{4}\right\rangle$ is a graph state characterized by its four stabilizer generators $X Z Z Z, Z X I I$, $Z I X I, Z I I X$. We may apply Hadamard transformations on all the qubits except the first one, the generators then become $K_{1}=X X X X, K_{2}=Z Z I I, K_{3}=Z I Z I, K_{4}=$ $Z I I Z$. In Pauli matrix form, we have $\left|G H Z_{4}\right\rangle\left\langle G H Z_{4}\right|=$ $\frac{1}{16}(I I I I+Z Z I I+Z I Z I+Z I I Z+I Z Z I+I Z I Z+I I Z Z$ $+Z Z Z Z+X X X X-Y Y X X-Y X Y X \quad-Y X X Y$ $-X Y Y X-X Y X Y-X X Y Y+Y Y Y Y)$. The noisy GHZ state is $\rho=p\left|G H Z_{4}\right\rangle\left\langle G H Z_{4}\right|+\frac{1-p}{16} I_{16}$. The biseparability and full separability of $\rho$ are known [9]. For the triseparability, we find the matched witness with parameters $M_{3300}=M_{3030}=M_{3003}=M_{0330}=M_{0303}=M_{0033}=$ $0, M_{3333}=2, M_{1111}=M_{2222}=1, M_{2211}=M_{2121}$ $=M_{2112}=M_{1221}=M_{1212}=M_{1122}=-1$. In the following, we will show that $M_{0000}$ is -2 . The critical $p$ for tri-separable is $p=\frac{1}{5}$. The state $\rho$ is a mixture of three part product states if $p \leq \frac{1}{5}$ and can not be three partite separable for $p>\frac{1}{5}$.

\subsection{The necessary condition}

Consider the qubits $1,2,3,4$, we first classify the qubits into three parts with the first two qubits in a part, the third and the fourth are the other two parts. We denote the partition as $12|3| 4$. For the given $M_{k l m n}$ except $M_{0000}$ in the main text, we have $-M_{0000}=\max f$, where $f=$ $2 T_{33} z_{3} z_{4}+\left(T_{11}+T_{22}\right)\left(x_{3} x_{4}+y_{3} y_{4}\right)+\left(T_{21}+T_{12}\right)\left(y_{3} x_{4}+\right.$ $\left.x_{3} y_{4}\right)$, subject to $x_{i}^{2}+y_{i}^{2}+z_{i}^{2}=1(i=3,4)$ and $T_{i j}=$ $\left\langle\psi\left|\sigma_{i} \otimes \sigma_{j}\right| \psi\right\rangle$, where $|\psi\rangle=\alpha|00\rangle+\beta|01\rangle+\gamma|10\rangle+\delta|11\rangle$ is a two qubit pure state. Then $T_{33}=|\alpha|^{2}+|\delta|^{2}-\left(|\beta|^{2}+|\gamma|^{2}\right)$, $T_{11}+T_{22}=2\left(\beta \gamma^{*}+\gamma \beta^{*}\right), T_{21}+T_{12}=2 i\left(\alpha \delta^{*}-\delta \alpha^{*}\right)$. Denote $a=z_{3} z_{4}, b=x_{3} x_{4}+y_{3} y_{4}, c=y_{3} x_{4}+x_{3} y_{4}$. The extremal values of $f$ are $a \pm 2 c=2 z_{3} z_{4} \pm 2\left(y_{3} x_{4}+x_{3} y_{4}\right) \leq 2$ and $-a \pm 2 c=-2 z_{3} z_{4} \pm 2\left(x_{3} x_{4}+y_{3} y_{4}\right) \leq 2$. The maximum of $f$ is 2 . Thus $f \leq 2$ for $12|3| 4$ partition. By the symmetry of the problem, we have $f \leq 2$ for all 6 kinds of partitions. Thus we have $M_{0000}=-2$, the necessary condition for tri-separablity is obtained as $p \leq \frac{1}{5}$.

\subsection{The sufficient condition}

The noisy four qubit $G H Z$ state with $p=\frac{1}{5}$ can be written as $\rho=\frac{1}{16}\left[\frac{1}{5}(I I I I+X X X X+Z Z I I-Y Y X X)+\frac{1}{5}(I I I I+\right.$ $Y Y Y Y-Y X Y X+I Z I Z)+\frac{1}{5}(I I I I-Y X X Y-X X Y Y+$ $Z I Z I)+\frac{1}{5}(I I I I-X Y Y X-X Y X Y+I I Z Z)+\frac{1}{5}(I I I I+$ $Z I I Z+I Z Z I+Z Z Z Z)]$. Each round bracket in the above expression is tri-separable. The first four round brackets are tri-separable in the following partitions respectively, $12|3| 4,13|2| 4,1|3| 24,1|2| 34$. The last one is fully separable. For example, the first round bracket is $(I I I I+X X X X+$ $Z Z I I-Y Y X X)=\frac{1}{2}(I I+Z Z+X X-Y Y)(I I+X X)$ $+\frac{1}{2}(I I+Z Z-X X+Y Y)(I I-X X)$. The components $(I I \pm X X)=\frac{1}{2}(I+X)(I \pm X)+\frac{1}{2}(I-X)(I \mp X)$ is separable for the third and the fourth qubits. The components $[I I+$ $Z Z \pm(X X-Y Y)]$ is proportional to valid two qubit states for the first two qubits. Thus $I I I I+X X X X+Z Z I I$ $-Y Y X X$ is tri-separable for partition $12|3| 4$. 


\section{The full separability of noisy four qubit cluster state}

The four qubit cluster state $\left|C l_{4}\right\rangle$ is a graph state characterized by its four stabilizer generators $K_{1}=X Z I I$, $K_{2}=Z X Z I, K_{3}=I Z X Z, K_{4}=I I Z X$, where $X, Y, Z$ are the Pauli matrices. The noisy cluster state is

$$
\rho=p\left|C l_{4}\right\rangle\left\langle C l_{4}\right|+\frac{1-p}{16} I_{16} .
$$

The biseparability of the state is known $[9$. We will consider the full separability of the state in this section and the three partite separablilty in the next section. The cluster state can be written as $\left|C l_{4}\right\rangle\left\langle C l_{4}\right|=\frac{1}{16} \prod_{j=1}^{4}\left(I+K_{j}\right)=$ $\frac{1}{16} \sum_{i_{1}, i_{2}, i_{3}, i_{4}=0}^{1} K_{4}^{i_{4}} K_{3}^{i_{3}} K_{2}^{i_{2}} K_{1}^{i_{1}}$. In the form of Pauli matrices, we have $\left|C l_{4}\right\rangle\left\langle C l_{4}\right|=\frac{1}{16}(I I I I+X Z I I+Z X Z I$ $+Y Y Z I+I Z X Z+X I X Z+Z Y Y Z-Y X Y Z+I I Z X$ $+X Z Z X+Z X I X+Y Y I X+I Z Y Y+X I Y Y-Z Y X Y+$ $Y X X Y)$. The characteristic function $R_{k l m n}$ of $\rho$ has the values of $1, p,-p$ or 0 . The number of nonzero $R_{k l m n}$ is 16 . The matched witness has 16 parameters $M_{k l m n}$.

For the full separability, we find that the witness with the following parameters is a matched witness, $M_{3130}=$ $M_{0313}=M_{3101}=M_{1013}=M_{2230}=M_{0322}=M_{2201}=$ $M_{1022}=1, M_{3223}=M_{1331}=M_{2112}=2, M_{2123}=$ $M_{3212}=-2, M_{1300}=M_{0031}=0$. In the following we will show that $M_{0000}$ is -2 . Hence, the noise tolerance of full separability for $\left|C l_{4}\right\rangle$ is $p_{t o l}=1-p$ with $p=\frac{1}{9}$. The state $\rho$ is fully separable for $p \leq \frac{1}{9}$ and is entangled for $p>\frac{1}{9}$.

\subsection{The necessary condition}

For the given $M_{k l m n}$ except $M_{0000}$, we have $-M_{0000}=$ $\max f$, where $f=\left\{\left(z_{1} x_{2}+y_{1} y_{2}\right)\left(z_{3}+x_{4}\right)+\left(z_{2}+x_{1}\right)\left(x_{3} z_{4}+\right.\right.$ $\left.y_{3} y_{4}\right)+2\left(z_{1} y_{2} y_{3} z_{4}+x_{1} z_{2} z_{3} x_{4}+y_{1} x_{2} x_{3} y_{4}-y_{1} x_{2} y_{3} z_{4}-\right.$ $\left.\left.z_{1} y_{2} x_{3} y_{4}\right)\right\}$, subject to $x_{i}^{2}+y_{i}^{2}+z_{i}^{2}=1(i=1, \ldots, 4)$. Using $x_{4}^{2}+y_{4}^{2}+z_{4}^{2}=1$, the maximization over $\left(x_{4}, y_{4}, z_{4}\right)$ leads to $f \leq f_{1}$ where

$$
f_{1}=b \cos \theta+\sqrt{(b+d \cos \theta)^{2}+e^{2} \sin ^{2} \theta} .
$$

With $b=z_{1} x_{2}+y_{1} y_{2}, \quad d=2 x_{1} z_{2}, \quad e=$ $\sqrt{\left(z_{2}+x_{1}\right)^{2}+4\left(z_{1} y_{2}-y_{1} x_{2}\right)^{2}}$, and $z_{3}=\cos \theta$ is assumed. There are two solutions for the maximization of $f_{1}$ over $\theta$. The first is $\sin \theta=0$, thus $f_{1}= \pm b+| \pm b+d| \leq 2$. The inequality comes from $f_{1}= \pm 2 b+d=2\left[ \pm\left(z_{1} x_{2}+y_{1} y_{2}\right)+x_{1} z_{2}\right] \leq 2$ when $\pm b+d \geq 0$ and $f_{1}=-d \leq 2$ when $\pm b+d<0$. The second solution is $\cos \theta=\frac{\bar{b}}{e-d}$ subject to $-1 \leq \frac{b}{e-d} \leq 1$. Thus $f_{1}=\frac{b^{2}}{e-d}+e$. A simple numeric calculation shows that $f_{1} \leq 2$. Hence $M_{0000}=-2$. The necessary condition of full separability is $p \leq \frac{1}{9}$.

\subsection{The sufficient condition}

The maximization of $f$ in above subsection hints the process of decomposing a separable state into its explicit separable expression. If the maximal $f_{1}=2$ is achieved by one of the terms, say $2 z_{1} y_{2} y_{3} z_{4}$, we have $2 z_{1} y_{2} y_{3} z_{4}=2$, The solutions of $z_{1} y_{2} y_{3} z_{4}=1$ are that $z_{1}, y_{2}, y_{3}, z_{4}$ should be equal to \pm 1 , the number of -1 should be odd. Then there are 8 solutions. Each solution corresponds to a product state, for example $z_{1}=y_{2}=y_{3}=z_{4}=1$ corresponds to a product state proportional to $(I+Z)(I+Y)(I+Y)(I+Z)$. Summing up all the 8 product states gives rise to unnormalized fully separable state $I I I I+Z Y Y Z$. Similarly, all the other solutions of $f=2$ can be utilized to obtained the product states. Thus the mixture of the product states will compose the noisy cluster state if the noise is under some threshold.

The noisy cluster state with $p=\frac{1}{9}$ can be written as $\rho=\frac{1}{16}\left[\frac{1}{9}(I I I I+Z Y Y Z)+\frac{1}{9}(I I I I+Y X X Y)+\frac{1}{9}(I I I I-\right.$ $Y X Y Z)+\frac{1}{9}(I I I I-Z Y X Z)+\frac{1}{9}(I I I I+Z X Z I+Z X I X+$ $I I Z X)+\frac{1}{9}(I I I I+Y Y Z I+Y Y I X+I I Z X)+\frac{1}{9}(I I I I+$ $I Z X Z+X I X Z+X Z I I)+\frac{1}{9}(I I I I+I Z Y Y+X I Y Y+$ $\left.X Z I I)+\frac{1}{9}(I I I I-I I Z X-X Z I I+X Z Z X)\right]$.

Each round bracket in the above expression is fully separable.

\section{Noise tolerance of four qubit cluster state in three parties}

For the tri-separability, we find the witness to be $W=$ $c I I I I-Q$, where

$$
\begin{aligned}
Q= & -X Z I I-I I Z X+3 X Z Z X \\
& +Z X Z I+X I Y Y+3 Y X X Y \\
& +Y Y Z I+X I X Z+3 Z Y Y Z \\
& +Z X I X+I Z X Z-3 Z Y X Y \\
& +Y Y I X+I Z Y Y-3 Z Y X Y,
\end{aligned}
$$

and $c=5$. The entanglement is detected if $\operatorname{tr}(\rho W)<0$, which leads to $p>\frac{5}{21}$. Hence the state $\rho$ is a mixture of three part product states if $p \leq \frac{5}{21}$ and can not be three partite separable for $p>\frac{5}{21}$.

\subsection{The necessary condition}

For all four qubit tripartite separable state $\rho_{3 s e p}$, we should have $\operatorname{tr}\left(\rho_{3 \text { sep }} W\right) \geq 0$, that is

$$
c=\max _{\rho_{3 s e p}} \operatorname{tr}\left(\rho_{3 s e p} Q\right) .
$$

Consider the qubits 1,2,3,4, we first classify the qubits into three parts with the first two qubits in a part, the third and the fourth are the other two parts. We denote the partition as $12|3| 4$. The tripartite separable state for 
this partition is $\rho_{12|3| 4}=\sum_{i} p_{i} \rho_{12}^{(i)} \otimes \rho_{3}^{(i)} \otimes \rho_{4}^{(i)}$, where $p_{i}$ form a probability distribution. Without loss of generality, we consider the states in each party to be pure. The tripartite separable state is a mixture of pure product state $\left|\psi_{12}\right\rangle\left|\psi_{3}\right\rangle\left|\psi_{4}\right\rangle$. Then for the partition $12|3| 4$, we

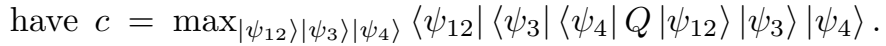
Then $c=\max _{\left|\psi_{12}\right\rangle}\left\langle\psi_{12}|\mathcal{M}| \psi_{12}\right\rangle$, where the matrix $\mathcal{M}=$ $\left\langle\psi_{3}\left|\left\langle\psi_{4}|Q| \psi_{3}\right\rangle\right| \psi_{4}\right\rangle$ is the partial trace of $Q$ over the third and the fourth qubits, respectively. Applying the Hadamard transform $\mathrm{H}_{2}$ on the first qubit, we obtain the matrix $\mathcal{M}^{\prime}=\left(H_{2} \otimes I\right) \mathcal{M}\left(H_{2} \otimes I\right)$, the eigenvalues do not change since the Hadamard transform is unitary. Hence $c$ is equal to the largest eigenvalue of $\mathcal{M}^{\prime}$. Denote the Bloch vectors of $\left|\psi_{i}\right\rangle$ as $\left(x_{i}, y_{i}, z_{i}\right)$ with $i=1, \ldots, 4$, it follows that, $\mathcal{M}^{\prime}=\left(3 z_{3} x_{4}-1\right) Z Z-z_{3} x_{4} I I+\left(x_{3} z_{4}+y_{3} y_{4}\right)(Z I+$ $I Z)+\left(z_{3}+x_{4}\right)(X X-Y Y)+3\left(y_{3} z_{4}-x_{3} y_{4}\right)(X Y+$ $Y X)$. The eigenvalues of $\mathcal{M}^{\prime}$ are $\lambda_{1,2}=2 z_{3} x_{4}-$ $1 \pm 2 \sqrt{\left(x_{3} z_{4}+y_{3} y_{4}\right)^{2}+\left(z_{3}+x_{4}\right)^{2}+9\left(y_{3} z_{4}-x_{3} y_{4}\right)^{2}}$, and $\lambda_{3,4}=1-4 z_{3} x_{4}$. The maximum of $\lambda_{1}$ is 5 and it is achieved when

$$
z_{3}=x_{4}=0, y_{3} z_{4}-x_{3} y_{4}= \pm 1
$$

or

$$
z_{3}=x_{4}= \pm 1 .
$$

The maximums of $\lambda_{3}$ and $\lambda_{4}$ are 5 and they are achieved when

$$
z_{3}=-x_{4}= \pm 1 \text {. }
$$

Hence the eigenvalues of $\mathcal{M}^{\prime}$ is tight upper bounded by 5 . We thus arrives $c=5$ for the partition $12|3| 4$.

Then we consider the partition $13|2| 4$, the first and the third qubits are in a party, the other two qubits are in the other two parties, respectively. We have $c=\max _{\left|\psi_{13}\right\rangle\left|\psi_{2}\right\rangle\left|\psi_{4}\right\rangle}\left\langle\psi_{13}\left|\left\langle\psi_{2}\left|\left\langle\psi_{4}|Q| \psi_{13}\right\rangle\right| \psi_{2}\right\rangle\right| \psi_{4}\right\rangle=$ $\max _{\left|\psi_{13}\right\rangle}\left\langle\psi_{13}|\mathcal{M}| \psi_{13}\right\rangle$, with matrix $\mathcal{M}=$ $\left\langle\psi_{2}\left|\left\langle\psi_{4}|Q| \psi_{2}\right\rangle\right| \psi_{4}\right\rangle$. Applying Hadamard transform to the first qubit and eliminating the phase factors of the matrix entries with unitary transformation, then the matrix $\mathcal{M}$ is transformed to $\mathcal{M}^{\prime}=U\left(H_{2} \otimes I\right) \mathcal{M}\left(H_{2} \otimes I\right) U^{\dagger}$, namely,

$$
\mathcal{M}^{\prime}=\left[\begin{array}{llll}
K_{1} & \alpha_{4} z_{2+} & \alpha_{2} x_{4+} & -3 \alpha_{2} \alpha_{4} \\
\alpha_{4} z_{2+} & K_{2} & 3 \alpha_{2} \alpha_{4} & \alpha_{2} x_{4-} \\
\alpha_{2} x_{4+} & 3 \alpha_{2} \alpha_{4} & K_{3} & \alpha_{4} z_{2-} \\
-3 \alpha_{2} \alpha_{4} & \alpha_{2} x_{4-} & \alpha_{4} z_{2-} & K_{4}
\end{array}\right] .
$$

Where $z_{2 \pm}=z_{2} \pm 1, x_{4 \pm}=x_{4} \pm 1, \alpha_{2}=\sqrt{1-z_{2}^{2}}, \alpha_{4}=$ $\sqrt{1-x_{4}^{2}}, K_{1,3}= \pm 3 z_{2} x_{4} \mp z_{2}-x_{4}, K_{2,4}=\mp 3 z_{2} x_{4} \mp z_{2}+x_{4}$. The unitary transformation for eliminating the matrix entry phase factors is $U=\operatorname{diag}\left\{1, e^{i \theta_{4}}, e^{-i \theta_{2}}, e^{i\left(\theta_{4}-\theta_{2}\right)}\right\}$, where $\theta_{2}=\tan ^{-1}\left(y_{2} / x_{2}\right), \theta_{4}=\tan ^{-1}\left(y_{4} / z_{4}\right)$. Thus . Let $t=x_{4}^{2} \alpha_{2}^{2}+z_{2}^{2} \alpha_{4}^{2}$, the eigenequation is $\lambda^{4}-2(11-8 t) \lambda^{2}+$ $8(4 t-3) \lambda+3(15-16 t)=0$, which can be factorized to $\left(\lambda^{2}+2 \lambda-3\right)\left(\lambda^{2}-2 \lambda+16 t-15\right)=0$, the maximal eigenvalue is $\lambda_{m}=1+4 \sqrt{1-t} \leq 5$. Hence we have $c=5$ for the partition $13|2| 4$. The maximal eigenvalue is achieved when $t=0$, namely

$$
x_{4}= \pm 1, z_{2}= \pm 1 ;
$$

or

$$
x_{4}=0, z_{2}=0 .
$$

For the partition $14|2| 3$, we have $c=$ $\max _{\left|\psi_{14}\right\rangle}\left\langle\psi_{14}|\mathcal{M}| \psi_{14}\right\rangle$, with matrix $\mathcal{M}=$ $\left\langle\psi_{2}\left|\left\langle\psi_{3}|Q| \psi_{2}\right\rangle\right| \psi_{3}\right\rangle$. We can transform the ma$\operatorname{trix} \mathcal{M}$ to $\mathcal{M}^{\prime}=U\left(H_{2} \otimes H_{2}\right) \mathcal{M}\left(H_{2} \otimes H_{2}\right) U^{\dagger}$, with $U=\operatorname{diag}\left\{1, e^{-i \theta_{3}}, e^{-i \theta_{2}}, e^{-i\left(\theta_{3}+\theta_{2}\right)}\right\}$, where $\theta_{3}=\tan ^{-1}\left(y_{3} / x_{3}\right)$. The $\mathcal{M}^{\prime}$ has the same form as in (27) with $z_{3}, x_{4}, \alpha_{3}, \alpha_{4}$ being substituted by $z_{2}, z_{3}, \alpha_{2}, \alpha_{3}$, respectively, where $\alpha_{2}=\sqrt{1-z_{2}^{2}}$. The maximal eigenvalue of $\mathcal{M}^{\prime}$ is 5 , hence $c=5$, which is achieved at

$$
z_{2}= \pm 1, z_{3}= \pm 1
$$

or

$$
z_{2}=0, z_{3}=0 .
$$

For the partition $1|23| 4$, we have $c=$ $\max _{\left|\psi_{23}\right\rangle}\left\langle\psi_{23}|\mathcal{M}| \psi_{23}\right\rangle$, with matrix $\mathcal{M}=$ $\left\langle\psi_{1}\left|\left\langle\psi_{4}|Q| \psi_{1}\right\rangle\right| \psi_{4}\right\rangle$. Using unitary transform $U=\operatorname{diag}\left\{1, e^{i \theta_{4}}, e^{i \theta_{1}}, e^{i\left(\theta_{1}+\theta_{4}\right)}\right\}$, with $\theta_{1}=\tan ^{-1}\left(y_{1} / z_{1}\right)$, we obtain $\mathcal{M}^{\prime}$ having the same form as in (27) with $z_{3}, \alpha_{3}$ being substituted by $x_{1}, \alpha_{1}$, respectively, where $\alpha_{1}=\sqrt{1-x_{1}^{2}}$. The maximal eigenvalue of $\mathcal{M}^{\prime}$ is 5 , hence $c=5$, which is achieved at

$$
x_{1}= \pm 1, x_{4}= \pm 1
$$

or

$$
x_{1}=0, x_{4}=0 .
$$

Since the witness is symmetric under exchange of the first qubit with the fourth, the second with the third, it follows that $c=5$ for the partitions $1|2| 34$ and $1|4| 23$. We have proven that $c=5$ for all six partitions.

\subsection{The sufficient condition}

For $p=\frac{5}{21}$, we will prove explicitly that the noisy cluster state (21) is tripartite separable. Let consider partition $1|23| 4$, the maximal eigenvalue of $\mathcal{M}$ is achieved for the conditions (32) or (33). The condition $x_{1}=1$, $x_{4}=1$ corresponds to the state $\frac{1}{2}(I+X)$ for the first qubit and $\frac{1}{2}(I+X)$ for the fourth qubit. The $x_{1}=1, x_{4}=1$ also leads to a diagonal $\mathcal{M}$. We have $\mathcal{M}=\operatorname{diag}\{1,-3,-3,5\}$. Hence the maximal eigenvalue is achieved by $\left|\psi_{23}\right\rangle=$ $|11\rangle$. Thus the pure state that achieves $c=5$ is $\frac{1}{4}(I+$ $X)|11\rangle\langle 11|(I+X)=\frac{1}{16}(I+X)(I-Z)(I-Z)(I+X)$. The other three cases of (32) lead to three similar separable states. Average on all these four state we arrive at the separable state

$$
\rho_{0}=\frac{1}{16}(I I I I-X Z I I-I I Z X+X Z Z X) .
$$


The condition (33) corresponds to states $\frac{1}{2}\left(I+\sin \theta_{i} Y+\right.$ $\left.\cos \theta_{i} Z\right)$ for the first $(i=1)$ and the fourth $(i=4)$ qubits. At this condition the eigenvector corresponds to the maximal eigenvalues is $\left|\psi_{23}\right\rangle=\frac{1}{4}\left(|00\rangle+e^{i \theta_{4}}|01\rangle+\right.$ $\left.e^{i \theta_{1}}|10\rangle-e^{i \theta_{1}+i \theta_{4}}|11\rangle\right)$. We may write $\left|\psi_{23}\right\rangle\left\langle\psi_{23}\right|=\frac{1}{4}(I I$ $+\cos \theta_{1} X Z+\sin \theta_{1} \sin \theta_{4} X X+\sin \theta_{1} Y Z+\cos \theta_{1} \cos \theta_{4} Y Y$ $+\cos \theta_{4} Z X-\cos \theta_{1} \sin \theta_{4} Y X+\sin \theta_{4} Z Y-\sin \theta_{1} \cos \theta_{4} X Y$ ). Denote the tripartite separable state as $\varrho_{1}\left(\theta_{1}, \theta_{4}\right)=$ $\frac{1}{4}\left(I+\sin \theta_{1} Y+\cos \theta_{1} Z\right)\left|\psi_{23}\right\rangle\left\langle\psi_{23}\right|\left(I+\sin \theta_{4} Y+\cos \theta_{4} Z\right)$. Let $\varrho_{1}=\frac{1}{16} \sum_{j, k=0}^{3} \varrho_{1}\left(\frac{(2 j+1) \pi}{4}, \frac{(2 k+1) \pi}{4}\right)$. Thus we have the tripartite separable state

$$
\bar{\varrho}_{1}=\xi+\frac{1}{32}(I Z X Z+I Z Y Y+Y Y Z I+Z X Z I),
$$

where $\xi=\frac{1}{16} I I I I+\frac{1}{64}(Y X X Y-Y X Y Z+Z Y Y Z-$ $Z Y X Y)$. Similarly, we have the tripartite separable states

$$
\begin{aligned}
& \bar{\varrho}_{2}=\xi+\frac{1}{32}(X I X Z+X I Y Y+Y Y I X+Z X I X), \\
& \bar{\varrho}_{3}=\xi+\frac{1}{32}(Z X Z I+X I Y Y+Y Y Z I+X I X Z), \\
& \bar{\varrho}_{4}=\xi+\frac{1}{32}(I Z X Z+Y Y I X+I Z Y Y+Z X I X),
\end{aligned}
$$

for partitions $14|2| 3,13|2| 4,1|3| 24$, respectively. We have a tripartite separable state

$$
\rho_{1}=\frac{1}{2}\left(\bar{\varrho}_{1}+\bar{\varrho}_{2}\right)=\frac{1}{2}\left(\bar{\varrho}_{3}+\bar{\varrho}_{4}\right) .
$$

For the partition $12|3| 4$, the maximal eigenvalue of $\mathcal{M}^{\prime}$ is achieved at the condition of either (24) or (25), or (26). When $z_{3}=x_{4}=1$, the third and the fourth qubits are in the states $\frac{1}{2}(I+Z)$ and $\frac{1}{2}(I+X)$, respectively. The $\mathcal{M}^{\prime}$ is reduced to $2 Z Z-I I+2(X X-Y Y)$ with eigenfunction $\frac{1}{\sqrt{2}}(|00\rangle+|11\rangle)$ for its largest eigenvalue $\lambda_{1}=5$. The corresponding eigenfunction for $\mathcal{M}$ is $\left|\psi_{12}\right\rangle=\frac{1}{2}(|00\rangle+$ $|01\rangle+|10\rangle-|11\rangle)$. Hence the tripartite separable state that achieves the condition $c=5$ is $\frac{1}{4}\left|\psi_{12}\right\rangle\left\langle\psi_{12}\right|(I+Z)(I+X)$ $=\frac{1}{16}(I I+X Z+Z X+Y Y)(I+Z)(I+X)$. Similarly, the tripartite separable state that achieves the condition $c=5$ is $\frac{1}{16}(I I+X Z-Z X-Y Y)(I-Z)(I-X)$ for the case $z_{3}=x_{4}=-1$. The average of these two states gives rise to the tripartite separable state $\varrho_{5}=\frac{1}{16}(I I I I+X Z I I+$ $I I Z X+X Z Z X+Z X Z I+Y Y Z I+Z X I X+Y Y I X)$. By the symmetry, we have the tripartite separable state $\varrho_{6}=\frac{1}{16}(I I I I+X Z I I+I I Z X+X Z Z X+I Z X Z+I Z Y Y+$ $X I X Z+X I Y Y)$ for the partition $1|2| 34$. We thus have a tripartite state

$$
\rho_{2}=\frac{1}{2}\left(\varrho_{5}+\varrho_{6}\right) .
$$

For the case of (24), $\mathcal{M}^{\prime}$ is reduced to $-Z Z \pm 3(X Y+Y X)$, where the sign \pm are for the cases $y_{3} z_{4}-x_{3} y_{4}= \pm 1$, respectively. The eigenvector for the largest eigenvalue $\lambda_{1}=5$ is $\frac{1}{\sqrt{2}}(|00\rangle \pm i|11\rangle)$. The corresponding eigenvector of $\mathcal{M}$ is $\left|\psi_{12}\right\rangle=\frac{1}{2}(|00\rangle \pm i|01\rangle+|10\rangle \mp i|11\rangle)$. Hence $\left|\psi_{12}\right\rangle\left\langle\psi_{12}\right|=$ $\frac{1}{4}[I I+X Z \pm(Y X-Z Y)]$. The third and the fourth qubits are $\frac{1}{2}\left(I+\cos \theta_{3} X+\sin \theta_{3} Y\right)$ and $\frac{1}{2}\left(I+\sin \theta_{4} Y+\cos \theta_{4} Z\right)$ with $\theta_{4}=\theta_{3} \pm \frac{\pi}{2}$, respectively. The product state of the third and the fourth qubits then is $\frac{1}{4}\left(I+\cos \theta_{3} X+\sin \theta_{3} Y\right)$ $\left(I \mp \sin \theta_{3} Z \pm \cos \theta_{3} Y\right)$. The average on $\theta_{3}=\frac{\pi}{4}, \frac{3 \pi}{4}, \frac{5 \pi}{4} \frac{7 \pi}{4}$ is the state of $\frac{1}{4}\left[I I \pm \frac{1}{2}(X Y-Y Z)\right]$. The tripartite separable states are $\frac{1}{16}[I I+X Z \pm(Y X-Z Y)]\left[I I \pm \frac{1}{2}(X Y-Y Z)\right]$. Averaging on the \pm states gives rise to a tripartite separable state $\varrho_{7}=\frac{1}{16}(I I+X Z) I I+\frac{1}{32}(Y X-Z Y)(X Y-Y Z)$. By the symmetry, for the partition $1|2| 34$, we have tripartite separable state $\varrho_{8}=\frac{1}{16} I I(I I+Z X)+\frac{1}{32}(Y X-Z Y)(X Y-$ $Y Z)$. Thus we have a tripartite separable state

$$
\rho_{3}=\frac{1}{2}\left(\varrho_{7}+\varrho_{8}\right) .
$$

At last, we can compose the tripartite separable state as

$$
\begin{aligned}
\rho & =\frac{1}{21}\left(\rho_{0}+12 \rho_{1}+4 \rho_{2}+4 \rho_{3}\right) \\
& =\frac{5}{21}\left|C l_{4}\right\rangle\left\langle C l_{4}\right|+\frac{1}{21} I I I I .
\end{aligned}
$$

\section{Conclusion}

We have utilized the characteristic coeffients (variables) of witness operator to investigate the multipartite separability of multipartite quantum states. The necessary condition of separability can be obtained for any given set of characteristic variables as far as the algebraic maximization can be worked out. The sufficient criterion is obtained by matching the characteristic variables to the given quantum state whose separability is under researched. We use the three qubit $\mathrm{X}$ shaped state to illustrate the process of finding the necessary and sufficient criterion of full separability with our method. New results are the necessary and sufficient conditions for tripartite separability and full separability of four qubit cluster state in white noise, the necessary and sufficient conditions for the tripartite separability of four qubit GHZ state in white noise. The noise tolerances of the tripartite separability and full separability of four qubit cluster state are $\frac{16}{21}$ and $\frac{8}{9}$, respectively. The noise tolerance of the tripartite separability of four qubit GHZ is $\frac{4}{5}$. These conditions are necessary and sufficient. We also explicitly construct the separable states for these four qubit noisy states with given parties. The matched witness method is suitble in finding the multipartite separable criterion for quatum states with less characteristic variables.

\section{Acknowledgment}

Supported by the National Natural Science Foundation of China (Grant Nos. 11375152) and (partially) supported 
by National Basic Research Program of China (Grant No. 2014CB921203) are gratefully acknowledged.

\section{References}

[1] R. F. Werner, Phys. Rev. A 40, 4277(1989).

[2] A. Peres, Phys. Rev. Lett. 77, 1413 (1996).

[3] M. Horodecki, P. Horodecki, and R. Horodecki, Phys. Lett. A 223, 1 (1996).

[4] O. Rodolph, Lett. Math. Phys. 70, 57 (2004).

[5] K. Chen and L.-A. Wu, Quantum Inf. Comput. 3, 193 (2003).

[6] O. Gühne, Phys. Rev. Lett. 92, 117903 (2004).

[7] A. C. Doherty, P. A. Parrilo and F. M. Spedalieri , Phys. Rev. Lett. 88 (2002)187904.

[8] M. Li, J. Wang, S.-M. Fei and X. Li-Jost, Phys. Rev. A 89, 022325 (2014).

[9] O. Gühne and M. Seevinck, New J. Phys. 12 (2010) 053002 .

[10] B. Jungnitsch, T. Moroder and O. Gühne, Phys. Rev. Lett. 106 (2011) 190502.

[11] B. M. Terhal, Phys. Lett. A 271, 319 (2000).

[12] O. Gühne, Phys. Lett. A 375 (2011) 406.

[13] A. Kay, Phys. Rev. A 83 (2011) 020303(R).

[14] X. Y. Chen, L. Z. Jiang, P. Yu, M. Tian, Quantum Inf Process 14 2463(2015).

\section{Appendix}

\section{A. Proof of Lemma 1.}

Proof: It is not difficult to eliminate two of the angles in $g(\varphi)$, say, $\varphi_{1}$ and $\varphi_{2}$ by maximization. We have

$$
g(\varphi) \leq \sqrt{A}+\sqrt{B},
$$

with $A=\delta^{2}+\gamma^{2}-2 \delta \gamma \cos 2 \varphi_{3}, B=\alpha^{2}+\beta^{2}+2 \alpha \beta \cos 2 \varphi_{3}$. The solutions of $\frac{d g_{1}\left(\varphi_{3}\right)}{d \varphi_{3}}=0$ are (i) $\sin 2 \varphi_{3}=0$, it gives rise to the second line of (9), and (ii)

$$
\cos 2 \varphi_{3}=\frac{\alpha^{2} \beta^{2}\left(\delta^{2}+\gamma^{2}\right)-\gamma^{2} \delta^{2}\left(\alpha^{2}+\beta^{2}\right)}{2 \alpha \beta \gamma \delta(\gamma \delta+\alpha \beta)},
$$

it gives rise to the first line of (9). The condition for the existence of the solution (ii) is $\left|\cos 2 \varphi_{3}\right| \leq 1$, which leads to $q \geq 0$. The condition $\delta \alpha \beta \gamma>0$ comes from $q_{0}^{2}=(\delta \alpha+\beta \gamma)(\delta \beta+\alpha \gamma)(\delta \gamma+\alpha \beta)-\delta \alpha \beta \gamma M_{111}^{2} \geq 0$. Thus when $\delta \alpha \beta \gamma>0$, the first line of (9) is larger than the second line.

B. Proof of (16)

We start from Eq. (13). The aim is to maximize $F_{2}\left(\theta_{1}, \theta_{2}\right)$ when $a=M_{1} c_{1}, b=M_{2}+M_{3} c_{1}, c=M_{3}+M_{2} c_{1}$, $d=M_{1}+M_{0} c_{1}, e=g_{m} s_{1}$ with $c_{1}=\cos \theta_{1}, s_{1}=\sin \theta_{1}$ and $g_{m}$ is related with $M_{i}$ as in the main text. The maximization of $F_{2}$ with respect to $\theta_{2}$ leads to two solutions. The first solution is $\sin \theta_{2}=0$, thus for $F_{3}\left(\theta_{1}\right)=$ $\max _{\theta_{2}} F_{2}\left(\theta_{1}, \theta_{2}\right)$, we have

$$
F_{3}\left(\theta_{1}\right)=a+b+|c+d|,
$$

which is a linear function of $c_{1}$. The maximal value is $F_{4}=$ $-M_{0}$ when $-M_{0}$ is positive and large enough. The second solution is

$$
\cos \theta_{2}=\frac{1}{e^{2}-d^{2}}\left(c d+b \sqrt{\frac{e^{2}\left(c^{2}+e^{2}-d^{2}\right)}{\left(b^{2}+e^{2}-d^{2}\right)}}\right) .
$$

Notice that the second solution does not exist if the absolute of right hand side of (46) exceeds 1 . The maximum of $F_{2}\left(\theta_{1}, \theta_{2}\right)$ with respect to $\theta_{2}$ is

$$
\begin{aligned}
F_{3}\left(\theta_{1}\right)= & a+\frac{1}{e^{2}-d^{2}}\left(b c d+\operatorname{sign}\left(b^{2}+e^{2}-d^{2}\right)\right. \\
& \left.\times \sqrt{e^{2}\left(c^{2}+e^{2}-d^{2}\right)\left(b^{2}+e^{2}-d^{2}\right)}\right) .
\end{aligned}
$$

The equation can be rewritten as

$$
e^{4}+\left(c^{2}+b^{2}-d^{2}-h^{2}\right) e^{2}+(h d-b c)^{2}=0 .
$$

where $h=a-F_{3}\left(\theta_{1}\right)$. Suppose $\left.F_{3}\left(\theta_{1}\right)\right|_{\theta_{1}=\theta_{0}}=-M_{0}$ for some $\theta_{0}$, then the solution of (48) is $\left.\cos \theta_{1}\right|_{\theta_{1}=\theta_{0}}=$ $\frac{-A+\sqrt{A^{2}-B^{2}}}{B}$, where $A=M_{0}^{2}+M_{1}^{2}-M_{2}^{2}-M_{3}^{2}, B=$ $2\left(M_{0} M_{1}-M_{2} M_{3}\right)$. Thus $-M_{0}$ is an achievable value of function $F_{3}\left(\theta_{1}\right)$. The derivative of Eq. (48) with respect to $x=\cos \theta_{1}$ at gives rise to $\left.\frac{d h}{d x}\right|_{x=x_{0}}=M_{1}$ and $\left.\frac{d^{2} h}{d x^{2}}\right|_{x=x_{0}}=-\left.\frac{A^{2}-B^{2}}{e^{2} h}\right|_{\theta=\theta_{0}}$, where $x_{0}=\cos \theta_{0}$. Hence we arrive at

$$
\begin{aligned}
\left.\frac{d F_{3}}{d x}\right|_{x=x_{0}} & =0, \\
\left.\frac{d^{2} F_{3}}{d x^{2}}\right|_{x=x_{0}} & =\left.\frac{A^{2}-B^{2}}{e^{2} h}\right|_{\theta=\theta_{0}}<0 .
\end{aligned}
$$

The inequality comes from the fact that $h\left(\theta_{0}\right)=M_{0}+$ $M_{1} \cos \theta_{0}<0$ if we choose $\left|M_{0}\right|>\left|M_{1}\right|$ and $M_{0}$ is negative. In order to make each of $m_{i}(i=0, \ldots, 3)$ positive, we have to choose $M_{0}$ with such a property. Combining all of the solutions together, we conclude that the maximum of $F_{2}\left(\theta_{1}, \theta_{2}\right)$ at case (ii) is $-M_{0}$ for sufficiently large and positive $-M_{0}$. 